\title{
DU RESPECT DES DROITS FONDAMENTAUX DE L'HOMME DANS LA PRISON DE KAMINA
}

\author{
Par KISEYA BWANA, Assistant à l'Université de Kamina
}

\section{INTRODUCTION}

Autant qu'il n'existe pas des sociétés sans crime parce que la criminalité est un phénomène permanent et universel, autant qu'il n'existe pas des sociétés qui ne répriment pas les crimes car ils constituent des actes proscrits. Ainsi, il existe de part le monde, une pléthore des mécanismes pour réprimer les crimes parmi lesquels le prononcé de la peine dans un jugement ou arrêt contre l'infracteur. En République Démocratique du Congo, les peines sont réglementées dans le code pénal et ne sont pas toutes exécutées de la même manière. Parmi les différentes peines, celles qui sont plus dissuasives sont exécutées en prison. Celle-ci n'est pas définie par le législateur congolais mais, ce dernier se borne à l'article 9 de l'ordonnance 344 du 17 septembre 1965 portant régime pénitentiaire à déterminer les catégories des personnes admissibles dans des prisons en ces termes : « Les prisons sont destinées à recevoir :

$1^{\circ}$ Les individus condamnés par un jugement ou arrêt coulé en force de la chose jugée :

a) A la peine de mort;

b) A une peine de servitude pénale principale;

c) A une peine de servitude pénale subsidiaire.

$2^{\circ}$ les individus mis à la disposition du gouvernement par une décision devenue définitive prise :

a) En application du $\S 6$ de la section II du livre I du code pénal;

b) En application des articles 3 et 4 du décret du 23 mai 1896 modifié par les décrets du 11 juillet 1923 et du 6 juin 1958 sur le vagabondage et la mendicité

$3^{\circ}$ Les personnes mises à la contrainte par corps :

a) En application de l'article 17 du livre I du code pénal;

b) En applications des articles 195 et suivants de l'annexe I à la loi du 10 juillet 1963 portant les dispositions relatives à l'impôt sur les revenus $»^{1}$.

La prison de nos jours est bien différente de celles de l'avant seconde guerre mondiale. En effet, depuis 1945, de nombreux changements ont été institués dans les prisons. La réforme impose une politique de resocialisation du condamné. Depuis cette date, des nombreuses modifications ont suivi, permettant d'améliorer toujours plus les conditions de vie dans les prisons ainsi que l'accompagnement des détenus pendant et après la détention. De nos jours, la réinsertion des détenus est devenue une préoccupation importante pour les communautés. Avec cette nouvelle conception de la prison, les prisonniers qui s'y trouvent n'ont pas seulement des devoirs mais aussi des droits à respecter par l'Etat qui prive la liberté en instituant les systèmes pénal et pénitentiaire. Notre réflexion dans cette œuvre scientifique 
consiste à faire l'état de lieu des droits fondamentaux de l'homme dans la prison de Kamina.

\section{PRESENTATION, HISTORIQUE ET FONCTIONNEMENT DE LA PRISON DE KAMINA}

La prison centrale de Kamina est située au Katanga, dans le ressort du district du Haut Lomami, territoire de Kamina au croisement des avenues Mobutu et K.D.L, tout près du camp de la police nationale congolaise, en face de l'hôpital Saint Eloi de la société nationale de chemin de fer du Congo. A défaut d'archives relatives à l'historique détaillé de la prison de Kamina, nous avons pu recueillir quelques données fournies par certaines notabilités de la place. En effet, l'actuelle prison de Kamina, a été construite vers les années 1930 dans le but d'y conserver les matériels de la société nationale de chemin de fer du Congo avant d'être convertie en établissement pénitentiaire à la suite d'une expropriation pour cause d'utilité publique. N'étant pas conçu pour cette fin, ce bâtiment vieux d'une quatre-vingtaine d'années compte sept dortoirs disposés à loger les détenus. Il comprend 3 bureaux pour l'administration pénitentiaire notamment : la direction, le greffe et le secrétariat, un petit local pour le dispensaire, trois entrepôts pour la garde du matériel et des vivres. A cela s'ajoute un cachot dans lequel sont placés les détenus punis selon les articles 77 à 80 de l'ordonnance 344 du 17 septembre 1965 relative au régime pénitentiaire. En outre, on y dénombre des locaux pour les installations hygiéniques et une cour intérieure d'environ 35 mètres carrés. Un quartier carcéral pour femmes, en annexe a été construit sur financement de la mission d'observation des nations unies pour la stabilisation de la paix au Congo en 2008. Le but de cette construction était de séparer les détenus hommes et femmes conformément à l'article 39 alinéa 2 de l'ordonnance précitée afin de prévenir les actes d'agression sexuelle car auparavant la cellule des femmes qu'on appelait Chicago était côte à côte de celles des hommes et plusieurs abus ont été enregistrés. Ce quartier est devenu opérationnel en 2013 après son équipement et inauguration.

Le bâtiment de la prison de Kamina est vétuste, inadapté et présente un aspect de délabrement très avancé. Les murs présentent des fissures, les tôles suintent, la charpente est pourrie et devient alors un danger permanent pour les détenus et le personnel pénitentiaire. Voici comment se présentent ces dortoirs et leurs dimensions

- La cellule Belgique mesure 40 mètres carrés;

- La cellule U.R.S.S mesure 28 mètres carrés;

- La cellule Chine mesure 20 mètres carrés;

- La cellule Ethiopie mesure 24 mètres carrés;

- La cellule Iran Irak mesure 32 mètres carrés;

- La cellule Soweto mesure 40 mètres carrés;

- La cellule Chicago mesure 27 mètres carrés.

- Une douche homme, longueur 7,15 mètres, largueur 4,85 mètres;

- Une douche femme, longueur 3, 45 mètres, largeur 3,20 mètres. 
La prison de Kamina a un personnel hiérarchisé comme suit : Le directeur de la prison, le directeur adjoint de la prison, le greffier et le secrétaire de la prison.

- Le directeur de la prison

Il est le seul chef de l'établissement. Il centralise, contrôle; et coordonne toutes les activités de l'établissement pénitentiaire. Il est en relation avec l'extérieur.

- Le directeur adjoint

Il seconde le directeur de la prison dans l'exercice de ses attributions.

- Le greffier de la prison

Il s'occupe de l'écrou, de la levée d'écrou, de la proposition de la libération conditionnelle, du classement des dossiers individuels des détenus, contact avec les différentes juridictions pour la régularisation des détentions irrégulières.

- Le secrétaire de la prison

Il est chargé de toutes les activités du secrétariat, c'est-à-dire qu'il s'occupe : de la rédaction, de la réception des correspondances, de l'expédition des correspondances, de l'établissement des procès verbaux, de la dactylographie, la détention, les registres d'évasion, de décès, de transfert, d'hospitalisation...

Il est important d'ores et déjà de signaler que le nombre du personnel pénitentiaire dans la prison de Kamina est très insuffisant, raison pour laquelle le poste d'intendant chargé de la gestion du patrimoine de la prison est assumé par le directeur de la prison. En outre, l'ordonnance 344 du 17septembre 1965 relative au régime pénitentiaire exige que le personnel de la prison soit des fonctionnaires de l'Etat. Il est curieusement et malheureusement constaté, qu'à la prison de Kamina, le surveillant portier est un condamné à une peine privative de liberté et cela crée une insécurité dans la mesure où ce condamné jouissant d'une large liberté d'entrée et de sortie, pourrait facilement s'y échapper. En ce qui concerne le corps de garde, il est constitué des policiers et des militaires qui sont installés dans la guérite située dans la cour de la prison. Ils ont pour mission de surveiller les entrées et les sorties de tout le monde dans la prison et de lutter contre les éventuelles évasions.

La capacité d'accueil d'une prison est le nombre total de détenus qu'elle peut héberger en respectant une surface minimale définie au préalable par personne ou par groupe de personnes. ${ }^{2}$ Etant construit comme un entrepôt de la société nationale de chemin de fer du Congo, il est vraiment difficile de déterminer précisément la capacité d'accueil de la prison de Kamina. Selon les estimations de certains experts de la mission d'observation des nations unies pour la stabilisation de la paix au Congo et le comité international de la croix rouge que nous avons consulté, cette prison ne peut détenir au delà de 120 détenus considérant sa surface totale de 408 mètres carrés. L'accentuation des crises politiques, économiques et sociales en République Démocratique du Congo avec comme conséquence l'augmentation permanente de la criminalité, Les arrestations arbitraires, l'insuffisance des magistrats au parquet de grande instance de Kamina pour traiter les dossiers dans le délai légal et l'absence d'audiences au Tribunal de Grande Instance de Kamina depuis juin 2013 par manque des juges, on assiste à une surpopulation carcérale. En date du 14 janvier 2014, la 
prison de Kamina contenait 190 détenus dont 187 hommes et 3 femmes parmi lesquels 166 prévenus et 24 condamnés.

\section{ANALYSE DE DROITS DES PRISONNIERS}

Bien qu'étant privé de la liberté, un détenu a des droits fondamentaux qui doivent être respectés. C'est ainsi que l'ordonnance 344 du 17 septembre 1965 relative au régime pénitentiaire prévoit certains droits en faveur des détenus notamment : le droit aux bonnes conditions d'incarcération, le droit à l'habillement décent, le droit aux soins médicaux, le droit à une nourriture équilibrée, le droit à la visite, le droit au travail et pécule. Dans cette partie de notre travail, nous ferons l'état de lieu de ces droits dans la prison de Kamina.

\section{II.1. Droits aux bonnes conditions d'incarcération}

Les conditions matérielles sont dégradantes, humiliantes et très éloignées des droits fondamentaux de l'homme dans la prison de Kamina. Ces réalités entrainent des nuisances de la prison sur les rôles qu'elle devait jouer. Outre donner la parole aux différents acteurs de ce monde, cette étude a collecté de nombreuses réponses des détenus fondant ainsi d'autant plus sa crédibilité car parler des conditions de vie ne peut se faire sincèrement qu'en donnant la parole aux délinquants qui ont vécu en prison, ou qui y vivent encore.

La première préoccupation est la très grande insatisfaction à l'unanimité des détenus que nous avons pu contacter quant à leurs conditions générales de détention. Une insatisfaction partagée par les professionnels du monde judiciaire. Dans ce mécontentement, nous pouvons distinguer les conditions de vie dans les cellules et les conditions de vie dans l'établissement.

Il est indispensable de préciser que la prison de Kamina n'a pas de cellules, mais au contraire des dortoirs qui étaient des pièces du bâtiment où on conservait les biens de la société nationale de chemin de fer du Congo. Le taux d'occupation ou la densité de la population carcérale est largement dépassé. Il est obtenu en rapportant le nombre de détenus présents à la date, au nombre de places définies par la capacité d'acceuil ${ }^{2}$. A titre illustratif, la prison de Kamina contenait 190 détenus en date du 14 janvier 2014 répartis de la manière suivante :

- Belgique : 40 détenus;

- URSS : 35 détenus;

- Chine : 5 détenus;

- Ethiopie : 29 détenus;

- Iran Irak : 37 détenus;

- Soweto : 41 détenus;

- Cellule carcérale femme : 3 détenus 
Le calcul de sa capacité d'accueil s'effectue comme suit: 190 multiplié par 100 divisé par $120=158,3 \%$. Donc, le taux de surpopulation en date du 14 janvier 2014 est de 58,3\%, c'est à dire $158,3-100$.

Si la capacité d'accueil est largement dépassée, il y'a surpopulation, les conditions de vie des détenus deviennent généralement difficiles. Quand il ya surpopulation, il y a aussi promiscuité qui peut d'ailleurs causer des problèmes graves à la santé des détenus et peut encore créer des évasions massives.

Outre la surpopulation carcérale chronique, les détenus ont à faire quotidiennement aux occupants permanents des dortoirs qui sont les poux, les punaises, les souris, les cancrelats, etc. Les toilettes et les douches sont en commun et ne laissent que peu de place à l'intimité. La prison n'a pas de cellules individuelles ou doubles.

Les détenus sont enfermés dans les dortoirs pendant toute la nuit et durant une partie de la journée. Ils restent dans les cours intérieures de 7H30' à $17 \mathrm{H} 00$ contraint de s'asseoir à même le sol comme à l'époque de l'antiquité parce que la prison ne dispose aucune chaise pour les détenus. L'allongement pour dormir, le déplacement dans les dortoirs, entreposage des effets personnels des détenus sont effectivement très difficile pour la simple raison que l'espace disponible pour recevoir un grand nombre de détenus fait défaut. Les dortoirs devraient en principe répondre aux exigences de superficie par personne. Les détenus sont entassés et ne disposent pas tous de sac de couchage si bien que bon nombre d'entre eux passent la nuit à même le sol. Les quelques couvertures qu'on y trouve proviennent des gens de bonne volonté qui viennent en aide aux prisonniers qui avec une natte, qui avec un sac, qui avec une couverture ${ }^{3}$. Faute des désinfectants et savons en permanence, ces couvertures sont très salles de telle sorte que quelques unes sont déclassées. Donc, les dortoirs de la prison de Kamina ne remplissent pas les conditions et ne répondent même pas aux normes de standard international.

A la porte d'entrée principale vers la coursive de la prison de Kamina, deux mentions y sont inscrites et attirent l'attention particulière de tout chercheur dans le domaine pénitentiaire. Ne dérogeant pas à cette observation en notre qualité de chercheur, nous avons aperçu toutes les deux mentions écrites en lingala (une des langues nationales de la République Démocratique du Congo) que nous avons analysé minutieusement. La première est hôtel KOTA OKOLA qui veut dire en français « hôtel où dès qu'on y entre, on grandi ». La deuxième est MEKA OSUKA NA ZAIRE qui veut dire en français « essaye et tu arriveras au bout au Zaïre ». Tous les efforts que nous avons fournis pour connaître la date à laquelle ces mentions ont été écrites et leurs auteurs se sont avérés vains car tous les agents de cette prison même les plus anciens ont échoué de nous donner une réponse exacte parce qu' ils ont trouvé ces mentions au moment de leur affectation dans la dite prison. Mais néanmoins, faisant une interprétation littérale et téléologique de ces mentions, nous en déduisons que c'est une façon de véhiculer le message aux futurs détenus que la prison de Kamina constitue un enfer.

Vivre dans la prison de Kamina constitue une agression permanente pour l'esprit et le corps. Ce sont les odeurs nauséabondes, des bruits, des voix fortes, des cris, une sensation 
pesante, oppressante de dureté dans les choses et les êtres. Les détenus sont obligés de souffrir de coups de chaleur. Imaginez-vous, arrivant dans une coursive d'une centaine des détenus qui vous scrutent, jaugent et menacent, une angoisse vous saisit à tel point que vous paralyse dans un mal être permanent dont il est difficile de s'en débarrasser.

La prison a ses règles, ses traditions, sa culture propre. C'est une micro société avec ses rites et ses lois aux antipodes de ce que l'on peut connaître à l'extérieur. Ici, la règle qui prédomine sur tout le reste est la loi du plus fort, du plus malin, plus vicieux. Il semble que tout ce qu'il y a de mauvais en l'homme, y soit réuni et tellement concentré que cela en dévient une matière qui se ressent, qui se palpe d'une atmosphère... une violence des mots, des coups des regards, des lieux, tout respire mal.

Les articles 49 et 50 de l'ordonnance 344 du 17 septembre 1965 relative au régime pénitentiaire disposent : « A leur entrée, les détenus passent à la douche. Leurs vêtements sont inspectés et subissent un traitement de désinfection; s'ils sont porteurs de parasites, ils sont traités à l'aide d'un produit adéquat ou placé dans une étuve.

Afin de permettre aux détenus de se présenter de manière convenable et de conserver le respect d'eux- mêmes, le règlement intérieur doit prévoir des mesures pour le bon entretien de la chevelure et de la barbe $»^{1}$.

Dans la prison de Kamina, le constat relatif aux soins corporels est trop amer. En effet, la prison de Kamina regorge en son sein des détenus préventifs et condamnés en provenance de tous les territoires du district du Haut Lomami à savoir : Kabongo, Bukama, Malemba Nkulu, Kaniama et Kamina. Vu les difficultés de transport aussi bien par voie routière que par voie ferrée, les détenus en provenance des territoires précités hormis parfois ceux de Kamina ville arrivent dans la prison très sales après qu'ils aient passés plusieurs jours de détention dans les maisons d'arrêt et pendant le voyage sans se laver, ni se débarbouiller. A leurs arrivés, il est incertain qu'ils passent directement à la douche de la prison du fait que le savon y fait souvent défaut. En outre, à l'entrée dans la prison, l'unique vêtement des détenus qui dégagent une odeur insupportable qu'ils ont porté durant la durée de la détention dans les maisons d'arrêt et le pèlerinage ne sont pas inspectés et par ricochet ne sont pas désinfectés s'ils sont porteurs de parasites par manque d'un agent engagé par l'Etat et affecté à cette fonction d'une part et par manque des désinfectants d'autre part.

Les agents de l'ordre public sont caractérisés par la brutalité au moment de l'arrestation, et les personnes arrêtées sont acheminées dans la prison en subissant des tortures. Ainsi, elles arrivent souvent en prison dépouillées de leurs chaussures. Les détenus qui n'ont pas des membres de famille proches ou des amis et connaissances intimes à Kamina qui peuvent leur donner les chaussures restent pieds nus dans la prison durant toute la journée même lorsqu'elles sont acheminées au parquet, à l'auditorat ou au tribunal.

L'article 48 l'ordonnance 344 du 17 septembre 1965 relative au régime pénitentiaire énonce : « chaque prison, chaque camp de détention et chaque maison d'arrêt doit disposer d'installations hygiéniques et, autant que possible, de douches et d'étuves à désinfecter. 
Le règlement d'ordre intérieur prescrit toutes les mesures relatives à la propreté et à l'entretien des locaux, des objets de couchage et des vêtements, ainsi qu'à la toilette des détenus $»^{1}$.

Le service pénitentiaire doit exiger des détenus la propreté personnelle. A cet effet, ils doivent disposer d'eau et des articles de toilette nécessaire à leur santé et à leur propreté. Afin de permettre aux détenus de se présenter de façon convenable et conserver le respect d'eux-mêmes, des facilités doivent être prévues pour le bon entretien de la chevelure et la barbe, les hommes doivent pouvoir se raser régulièrement. En effet, tous les prisonniers sont en droit d'être détenus dans les conditions décentes et humaines. C'est-à-dire les exigences d'hygiène adéquates constituent un critère d'appréciation du caractère décent et humain des conditions de détention.

Le médecin a la responsabilité importante de veiller au respect des normes d'hygiènes. A cet effet, il doit faire les inspections régulières et conseiller le directeur de la prison quant au caractère approprié des aliments, d'eau potable, de l'hygiène, de la propreté des installations sanitaires. Les installations sanitaires doivent permettre aux détenus de satisfaire au besoin naturel au moment voulu, d'une manière propre et descente. Les installations de bain et de douches doivent être suffisantes pour que chaque détenu puisse être à même et tenu de les utiliser, à une température adaptée au climat et aussi fréquemment que l'exige l'hygiène générale selon la saison et la raison géographique. Tous les locaux fréquentés régulièrement par les détenus doivent être maintenus en parfait état d'entretien et des propretés ${ }^{4}$.

A cet égard, force est de constater que l'inflation carcérale observée depuis ces dernières années, a entrainé une nette détérioration des conditions sanitaires au sein de la prison de Kamina. L'entassement en cellule observé, favorise la transmission des maladies contagieuses et le développement des troubles psychosomatiques, les locaux des douches et toilettes sont souvent insalubres, sans aération. L'utilisation intensive de ces équipements, du fait de la surpopulation, entraîne leur dégradation accélérée. Les détenus se contentent des douches et des toilettes collectives, avec risques afférents, comme la propagation de la gale ou le problème dermatologique. Le respect des normes élémentaires d'hygiènes fait vraiment défaut. Le puits de forage d'eau qui est dans l'enceinte de la prison n'est plus opérationnel depuis belle lurette. Cette situation déplorable et inconcevable entraine des difficultés sérieuses pour desservir la prison d'eau en permanence. De ce fait, dans les toilettes de la prison de Kamina les matières fécales sont de fois perceptibles faute d'eau en permanence pour les évacuer et cela occasionne la prolifération des microbes dans la prison. Le prisonnier vit son calvaire nuit et jour puisque jeté en pâture aux picotements dus aux morsures des bestiaux parasites, poux et punaises, que le service pénitentiaire ne détruit plus depuis la dernière livraison par le comité international de la croix rouge, des produits désinfectants en 2011. ${ }^{3}$ Pour bénéficier de l'eau du puits de fortune qui est creusé derrière la prison de Kamina, les détenus sont obligés de négocier moyennant paiement d'une modique somme d'argent à leurs collègues qui sont autorisés à faire le champ ou à préparer la nourriture dans l'enceinte de la prison, en sus, vu la vétuste des seaux de la prison de Kamina, tous sont troués et par conséquent déclassés. De ce fait, certains détenus disposent de leur 
propre seau qu'ils utilisent dans la prison pour des fins personnelles et ils les font de fois louer à d'autres détenus moyennant l'argent. Or, plusieurs détenus sont des vulnérables qui n'ont pas de pécunia dans la prison donc, ils sont contraints de passer plusieurs jours sans se laver.

La propreté de la bouche et des dents en particulier est une recommandation faite par les médecins dentistes à tout Homme qui en principe doit brosser ses dents chaque matin et après les repas avec le dentifrice pour éviter la prolifération des microbes dans la bouche. Dans la prison de Kamina, le brossage des dents par les détenus est une exception parce que plusieurs détenus n'ont pas des brosses à dent et par ricochet des dentifrices. Cette situation néfaste occasionne inéluctablement les problèmes de santé bucco-dentaire.

Les corps et les habits de plusieurs détenus dégagent une odeur nauséabonde et leurs bouches une mauvaise haleine. L'esprit de récolter mordicus les données fiables nous a caractérisé lors de nos entretiens avec les prisonniers dans leurs dortoirs, c'est la raison pour laquelle nous avons accepté d'affronter tous les aléas décrits si non, on devrait se limiter dans les bureaux.

\section{II.2. Droit à l'habillement décent}

Considérant le détenu comme tout être humain ayant droit de mener une vie descente nonobstant la privation de la liberté, les articles 51 et 52 de l'ordonnance 344 du 17 septembre 1965 disposent : « les détenus des prisons et des camps de détention sont revêtus d'une ténue. Le gardien peut prescrire que les détenus des maisons d'arrêt ou certaines catégories d'entre eux soient revêtus d'une ténue.

La ténue doit être appropriée au climat et suffisante pour maintenir le détenu en bonne santé

La tenue ne peut d'aucune manière être dégradante ou humiliante. Les vêtements doivent être maintenus constamment en bon état de propreté et d'entretien $»^{1}$.

L'habillement entant qu'élément du droit à un niveau de vie adéquat, figure parmi les droits de l'homme. Il faut fournir une ténue appropriée aux détenus qui ne sont pas autorisés à porter leurs vêtements personnels. Il faut que l'Etat prévoit les installations permettant d'assurer la propreté des vêtements et de les maintenir en bon état. Il faut des équipements permettant de procéder régulièrement au lavage et au séchage des vêtements.

Le paragraphe 1 de l'article 11 du pacte international relatif aux droits économiques, sociaux et culturels énonce le droit à l'habillement en tant qu'élément du droit de toute personne à un niveau de vie adéquat. L'ensemble des règles minima pour le traitement des détenus comporte les exigences suivantes :

- Tout détenu qui n'est pas autorisé à porter ses vêtements personnels doit recevoir un trousseau qui soit approprié au climat et suffisant pour le maintenir en bonne santé. Ces vêtements ne doivent en aucune manière être dégradants ou humiliants;

- Tous les vêtements doivent être changés et lavés aussi fréquemment qu'il est nécessaire le maintenir de l'hygiène; 
- Dans les circonscriptions exceptionnelles, quand le détenu s'éloigne de l'établissement à des fins autorisées, il doit lui être permis de porter ses vêtements personnels ou les vêtements n'attirant pas l'attention.

Lorsque les détenus sont autorisés à porter leurs vêtements personnels, des dispositions doivent être prises au moment de l'admission à l'établissement pour assurer que ceux-ci -soient propres et utilisables. Le médecin doit faire des inspections régulières et conseiller le directeur en ce qui concerne la qualité et la propreté des vêtements ${ }^{4}$. La prison de Kamina ne bénéficie pas des tenues en faveur de ses pensionnaires. En effet, il est triste de voir la façon dont les prisonniers de la prison centrale de Kamina sont vêtus. Chacun d'eux est habillé de sa manière, en vêtement qu'il portait dès le jour de son arrestation. Plusieurs vêtements de ces derniers sont à l'état débraillé, déchiqueté et de salubrité à cause du manque de tenues appropriés à eux et aussi à cause du manque des savons pour les laver. Cela prouve combien de fois ces prisonniers sont abandonnés.

Il y'a certains détenus qui au moment de leurs arrestations étaient habillés en tenue légère, sont restés avec ces habillements dans la prison durant toute la période de la détention peu importe la saison et la température. Ainsi, ils ont été exposés et frappés par diverses maladies causées par le froid notamment la pneumonie, la toux, le rhume, le rhumatisme. En revanche, d'autres détenus qui au moment de leurs arrestations étaient habillés en tenue lourde, sont restés avec ces habillements dans la prison durant toute la période de la détention, peu importe la saison et la température. Ainsi, ils ont été dérangés par l'étouffement.

L'Etat congolais est juridiquement responsable des diverses maladies précitées qui sont attrapées par les détenus dans la prison à cause de la violation des articles 51 et 52 de l'ordonnance 344 du 17 septembre 1965 portant régime pénitentiaire. Les initiatives privées pour soulager tant soi peu la misère des prisonniers méritent être signalées. En effet, certaines confessions religieuses, organisations non gouvernementales, la mission d'observation des nations unies pour la stabilisation de la paix au Congo et certains particuliers apportent occasionnellement des dons d'habits aux détenus vulnérables de la prison.

\section{II.3. DROIT AUX SOINS MEDICAUX}

La présence d'un dispensaire est obligatoire dans toutes les prisons car les détenus sont pris en charge par l'Etat conformément à l'article 59 alinéa 1 de l'ordonnance 344 du 17 septembre 1965 qui dispose : «Les détenus sont soignés au dispensaire ou à l'infirmerie de la prison, de la maison d'arrêt y annexée ou du camp de détention $»^{1}$.

La responsabilité essentielle des médecins, des infirmiers et de tout personnel médical travaillant en prison consiste à s'occuper de la santé des détenus. Majoritairement issue des milieux sociaux précarisés tant sur le plan social que sanitaire, la population carcérale se caractérise par une forte nécessité de soins, d'autant plus que le milieu clos de la prison provoque l'apparition ou l'aggravation de certaines affections et qu'il en découle des troubles pathologiques et sanitaires assez importants. 
L'infirmerie ou le dispensaire de la prison de Kamina est confronté à l'évolution sociologique de la population carcérale. L'administration des soins de santé aux détenus répond à certains préalables : les locaux adaptés à cette fin, le personnel compétent, le matériel adéquat, etc.

Le bâtiment abritant la prison de Kamina ne dispose pas des locaux adéquats pour son infirmerie parce qu'il n'était pas construit par son concepteur pour être une prison mais un entrepôt de la société nationale de chemin de fer du Congo. L'infirmerie de la prison de Kamina occupe un petit local mal aéré dans lequel on consulte, examine et administre les soins de santé aux détenus. Cette insuffisance des locaux défavorise une bonne administration des soins médicaux. L'article 59 alinéa 2 de l'ordonnance 344 du 17 septembre 1965 dispose : «Un quartier spécial destiné à recevoir les détenus atteints de maladies contagieuses sera aménagé dans les prisons, maisons d'arrêt y annexées et camps de détention $»^{1}$. Pour des raisons évoquées dans les lignes précédentes, la prison de Kamina ne dispose pas d'un quartier spécial destiné à recevoir les détenus atteints des maladies contagieuses. Mais néanmoins, l'ancien dortoir appelé communément dans la prison CHICAGO qui était jadis réservé aux détenues femmes avant la construction par la mission d'observation des nations unies pour la stabilisation de la paix en République Démocratique du Congo d'une cellule carcérale femme est utilisé faute d'un bâtiment approprié, comme quartier spécial pour l'incarcération des détenus déclarés atteints des diverses maladies contagieuses telles que lèpre, gale, mycose...Cette situation serait à la base de la contamination des microbes par les détenus à cause de la cohabitation ensemble car, lorsque les détenus atteints à titre illustratif de la tuberculose sont dans le même dortoir que ceux atteints de gales, ils pourraient réciproquement se contaminer les microbes c'est-à-dire, les détenus tuberculeux contamineraient la tuberculose aux détenus atteints de la gale et ces derniers pourraient contaminer leurs maladies aux tuberculeux.

L'article 54 alinéas 2 et 3 de l'ordonnance 344 du 17 septembre 1965 sur le régime pénitentiaire dispose : «Le gouverneur de province ou son délégué charge un médecin de desservir les prisons, camps de détention et maisons d'arrêt établis sur le territoire de la province.

Selon l'importance de la population pénitentiaire, le médecin visite l'établissement soit quotidiennement, soit une ou plusieurs fois par semaine $»^{1}$. Conformément à cette disposition, un médecin de l'hôpital général de référence de Kamina était désigné pour prester ses services en faveur des pensionnaires de la prison de Kamina. Selon les informations recueillies auprès du personnel pénitentiaire et des détenus, ce médecin était doué de conscience professionnelle parce qu'il s'acquittait positivement de ses obligations professionnelles au delà même des moyens mis à sa disposition. Mais cependant, depuis qu'il a effectué le voyage en juin 2014 vers Kinshasa jusqu'à ce jour (14 janvier 2015), malheureusement aucun médecin ne se présente à la prison pour s'occuper des soins médicaux en faveur des détenus qui ne savent à quel saint se voué. Tous les rapports et toutes les demandes dressés par le directeur de la prison à la hiérarchie afin d'affecter un autre médecin sont restés jusqu'à preuve du contraire lettre morte. L'absence d'un médecin pour desservir la 
prison de Kamina a des conséquences néfastes dans la mesure où elle prive les détenus des soins médicaux qui ne peuvent pas être administrés par les infirmiers mais uniquement par les médecins.

L'article 55 alinéas 2 et 3 de l'ordonnance 344 du 17 septembre 1965 sur le régime pénitentiaire dispose : « Le gouverneur de province ou son délégué affecte à chaque prison, camp de détention et maison d'arrêt établis sur le territoire de la province, selon l'importance de la population pénitentiaire, un ou plusieurs infirmiers ou infirmières.

Les infirmiers ou infirmières sont placés sous le contrôle et la surveillance technique du médecin, et sous le contrôle et la direction administratifs du gardien $»^{1}$. Les soins de santé dans l'infirmerie de la prison de Kamina sont assurés par deux infirmiers de l'hôpital général de référence de Kamina qui travaillent de 8 h00 à 11 h00 du lundi au samedi. En dehors de ses heures, si un cas de maladie se présentait, le personnel de la prison contacte par téléphone les deux infirmiers pour venir promptement afin de soigner le détenu malade. Si cette démarche n'aboutit pas parce que les infirmiers sont injoignables ou empêchés ou en cas d'urgence, le détenu malade est transféré toute affaire cessante à l'hôpital général de référence de Kamina.

Le dispensaire n'est ravitaillé ni en médicaments, ni moins encore en matériel. Comment voulez vous que les conditions sanitaires soient améliorées? Ainsi après la consultation et les examens médicaux, les infirmiers remettent les ordonnances médicales aux détenus malades qui doivent se débrouiller pour payer les produits pharmaceutiques. Pour les détenus dépourvus des moyens financiers, ces ordonnances médicales constituent des simples paperasses parce qu'ils ne serviront à rien. Ce constat funeste prouve à suffisance que la réglementation pénitentiaire concernant le droit aux soins médicaux en faveur des détenus de la prison de Kamina n'est pas respectée par l'Etat congolais. Les maladies les plus répandues en prison sont notamment le paludisme, la tuberculose, l'appendicite, l'hernie, la fièvre typhoïde, la gale, la mycose, la diarrhée. A cet effet, la population carcérale dans son ensemble ne bénéficie pas des soins de santé d'un niveau suffisant car l'administration des soins de santé faisant l'objet de la présente étude est dérisoire. L'inquiétude à celle-ci ne vient pas seulement de nous, mais aussi des acteurs judiciaires, comme le procureur de la République près le tribunal de grande instance, dans son rapport d'inspection du mois de février 2012 écrit : « la réfection de ce bâtiment permettra de remédier à la précarité des conditions hygiéniques et sanitaires $» .^{5}$

Les règles en matière des soins de santé dans la prison doivent impérativement faire l'objet d'une attention particulière par l'Etat. Il n'ya pas lieu de prétendre qu'en raison de son incarcération, une personne détenue est vouée à un plus bas niveau de soins de santé, par comparaison aux autres membres de la communauté. Le détenu conserve tous ses droits même en étant en prison, à part la liberté qui lui est retirée par une décision judiciaire. Ainsi, en privant un individu de sa liberté, l'Etat doit assumer sa responsabilité particulière qui consiste à lui assurer des soins de santé adéquats.

L'article 60 de l'ordonnance 344 du 17 septembre 1965 dispose : « si le médecin estime qu'en raison de la gravité ou de la nature de la maladie, il est impossible de soigner le déte- 
nu dans la prison, le camp de détention ou la maison d'arrêt, celui-ci est conduit à la formation médicale ou hospitalière la plus proche.

A la formation médicale ou hospitalière, le détenu est placé dans une chambre séparée; sa garde est assurée par la police locale.

Si le malade ainsi transféré est un prévenu, le gardien est tenu d'aviser du transfert, sur le champ, l'autorité judicaire et l'inspecteur territorialement compétent chargé de la direction de la section d'inspection des établissements pénitentiaires $»^{1}$.

Dans la ville de Kamina, si l'infirmier à défaut du médecin constate que la maladie du détenu est gravissime et qu'il n y'a plus moyen de le soigner au dispensaire de la prison, il sera transmis à l'hôpital général de référence de Kamina pour des soins appropriés. Le choix toujours de cet hôpital se justifie du fait que d'une part c'est un hôpital qui appartient à l'Etat et d'autre part, l'Etat est dans l'obligation de prendre en charge les soins médicaux de tout détenu peu importe la gravité.

\section{II.4. DROIT A UNE NOURRITURE EQUILIBREE}

Une alimentation adéquate et un approvisionnement suffisant en eau potable figurent parmi les droits humains. Tous les détenus ont droit à une nourriture saine et équilibrée aux heures normales du repas. En effet, l'article 11 du pacte international relatif aux droits économiques, sociaux et culturels garantit le droit à une alimentation adéquate entant que composante du Droit de toute personne à un niveau de vie adéquate.

Dans cet ordre d'idée l'article 61 alinéa 1 de l'ordonnance 344 du 17 septembre 1965 dispose : «Les détenus reçoivent une nourriture correspondant le plus possible à leur nourriture habituelle. Cette nourriture doit avoir une valeur suffisante pour maintenir le détenu en parfaite condition physique $»^{1}$.

Dans la mesure où l'Etat a privé les détenus de la possibilité d'assurer leurs propres besoins fondamentaux, ces derniers doivent être assurés par l'Etat. L'idéal serait que la vie en prison ne soit pas du tout différente de celle en milieu libre sous réserve de la privation de liberté. Une nourriture équilibrée dont doivent bénéficier les détenus est celle qui contient selon les experts, 15 à $20 \%$ de protéines, 40 à $45 \%$ d'hydrates de carbones, 30 à $35 \%$ de lipides. $^{6}$

L'article 203 alinéa 1 et point 5 de la constitution du 18 février 2006 telle que modifiée par la loi n $\mathrm{n}^{\circ} 11 / 002$ du 20 janvier 2011 proclame : « Sans préjudice des autres dispositions de la présente constitution, les matières suivantes sont de la compétence concurrente du pouvoir central et des provinces :

5. L'administration des cours et tribunaux, des maisons d'arrêt et de correction et des prisons pour peine $\gg^{7}$. A la lumière de cette disposition, la prise en charge des détenus est une obligation aussi bien du gouvernement central que du gouvernement provincial. De ce fait, dans tous les budgets nationaux et provinciaux qui se succèdent chaque année, il y'a une rubrique réservée aux dépenses des prisons parmi lesquelles celles de la prison de Kamina. Malheureusement depuis que le gouvernement provincial du Katanga avait été instal- 
lé en 2007 jusqu'à ce jour (14/01/2015), le gouvernement central a décliné ses responsabilités pour des raisons non élucidées en n'envoyant plus les frais de restauration des détenus. Ceci soulève la question de la destination des frais budgétisés par le gouvernement central pour la prison de Kamina. La réponse à cette question devrait être donnée par les ministères des finances, budget et justice et par les députés nationaux élus du haut lomami censés faire le suivi de l'exécution du budget national dans le haut lomami. Le gouvernement provincial du Katanga envoi chaque mois une somme d'argent en passant par le chef de division provinciale de la justice et garde des sceaux à Lubumbashi pour la prise en charge de la restauration des détenus de la prison de Kamina. Le montant exact ne nous a pas été révélé.

L'article 62 alinéas 1 et 2 de l'ordonnance 344 du 17 septembre 1965 sur le régime pénitentiaire dispose : « Les détenus font trois repas par jour.

Le gardien surveille ou fait surveiller la préparation et la distribution des aliments » ${ }^{1}$ L'alimentation est d'abord assurée par l'administration pénitentiaire, donc un repas par jour; ensuite par les familles des détenus qui résident non loin de la prison, enfin par différentes confections religieuses et par des personnes de bonne volonté de la place. Une cuisine collective est érigée à coté de la prison pour la préparation de la nourriture. Elle est ténue par les détenus sous la supervision d'un surveillant intendant. Ce dernier assiste à la préparation et la distribution des repas. En violation de l'ordonnance 344 du 17 septembre 1965 sur le régime pénitentiaire, les détenus de la prison de Kamina mangent une fois par jour soit le fufu et les feuilles de manioc ou le fufu et le haricot entre 13 hoo et $15 \mathrm{~h} 00$. Les feuilles de manioc et les haricots sont préparés en petite quantité et sans y mettre les épices. Cette nourriture habituelle n'a pas une valeur suffisante pour maintenir le détenu en parfaite condition physique. Les détenus qui pour une raison ou une autre ne mangent pas la nourriture préparée en prison sont abandonnés à leur triste sort.

Un autre aspect mérite d'être relevé, celui de la considération des besoins nutritifs de certains groupes vulnérables parmi lesquels figurent ceux qui suivent un traitement médical, les femmes allaitantes ou enceintes et les mineurs. Ces groupes reçoivent un repas supplémentaire à raison de leur vulnérabilité. Parmi les détenus de la prison de Kamina, certains devraient respecter un régime alimentaire spécial comme dans leur domicile tels que les hypertendus sont déconseillés à manger d'aliments salés, les cardiopates et les obèses sont interdits à consommer les aliments contenant beaucoup d'huile, les tuberculeux doivent manger à leur fin, les femmes qui allaitent doivent boire de Thé chaud au moins trois fois par jour pour faire couler le lait maternel... A cause de la modicité des frais de restauration, les régimes spéciaux des détenus pour telle ou telle autre raison ne sont pas respectés.

La question de savoir s'il faut encourager ou interdire les familles des détenus à leur fournir de la nourriture? D'une part, eu égard à la culture locale, les familles sont censées fournir de la nourriture par solidarité clanique. D'autre part, la quantité de la nourriture fournie par l'administration est insuffisante, donc les familles ne peuvent en aucun cas être interdites à apporter à manger aux détenus. Une pratique infractionnelle déplorable a élu domicile depuis belle lurette dans la prison de Kamina au vu et au su de tout le monde. Elle 
consiste pour les policiers et militaires commis à la sécurité de la prison d'exiger une somme d'argent illicite aux membres de la famille, amis et connaissances des détenus qui viennent leur donner la nourriture. Cette perception d'un droit illicite est constitutive d'infraction de concussion définie par AKELE ADAU Pierre comme : « la perception indue ou exagérée des droits, des taxes, faite sciemment par un fonctionnaire de l'Etat par abus de ses fonctions $»^{8}$. La base légale de la concussion est l'article $146 \mathrm{du}$ code pénal congolais. Cet article dispose : « Seront punis d'une servitude pénale de six mois à cinq ans tous fonctionnaires ou officiers publics, toutes personnes chargées d'un service public ou parastatal, toutes personnes représentant les intérêts de l'Etat ou d'une société étatique au sein d'une société privée, parastatale ou d'économie mixte en qualité d'administrateurs, de gérants, de commissaires aux comptes ou à tout autre titre, tous mandataires ou préposés des personnes énumérées ci-dessus qui se sont rendus coupables de concussion en ordonnant de percevoir, en exigeant ou en recevant ce qu'ils savaient n'être pas dû ou excéder ce qui était dû pour droits, taxes, contributions, revenus ou intérêts, pour salaires ou traitements, pour indemnités, primes ou tout autre avantage.

En condamnant à la peine prévue à l'alinéa précédent, le juge prononcera en outre :

$1^{\circ}$. La confiscation de la rétribution perçue par le coupable ou du montant de sa valeur lorsqu'elle n'a pu être saisie si la concussion résulte de la perception illicite, pour le compte du concussionnaire ou d'un tiers autre que l'Etat, des avantages inclus cités à l'alinéa précédent;

$2^{\circ}$. L'interdiction pour cinq ans au moins et dix ans au plus, après l'expiration de la peine, du droit de vote et du droit d'éligibilité;

$3^{\circ}$. L'interdiction d'accès aux fonctions publiques et paraétatiques quel qu'en soit l'échelon;

$4^{\circ}$. La privation du droit à la condamnation ou à la libération conditionnelle et à la réhabilitation dont le but est de faire bénéficier le coupable des avantages prohibés à l'article 145 de la présente section;

$5^{\circ}$. L'expulsion définitive du territoire de la République après l'exécution de la peine si le condamné est un étranger $»$.

Le taux du chiffre noir de la criminalité en rapport avec l'infraction de concussion commise par les policiers et militaires est trop élevé dans ce sens que les autorités militaires et policières sont au courant de cette situation mais elles ne font rien pour la découragée en infligeant des sanctions disciplinaires aux militaires et policiers concussionnaires. En outre, l'auditorat militaire de garnison qui est d'ailleurs opérationnel à Kamina ne s'est jamais saisi d'office de la concussion commise par les militaires et policiers afin qu'ils subissent des sanctions judiciaires prononcées par le tribunal militaire de garnison de Kamina.

Comme la prison dispose d'un terrain cultivable à côté, les détenus y travaillent. Il convient d'encourager cette initiative de l'administration, car elle assure l'approvisionnement alimentaire. Les détenus exploitent la terre et produisent leur propre nourriture telles que : maïs, haricot, arachide, patate douce, courge, tomate, choux...cette production parvient à suppléer tant soit peu à la ration du pouvoir public. Nous avons constaté inéluctable- 
ment que la plupart des pensionnaires de la prison de Kamina présentent un amaigrissement suite aux mauvais traitements qu'ils sont victimes en matière nutritive, cela constitue une violation notoire des droits à leur égard.

\section{II.5. DROIT AUX CONTACTS AVEC L'EXTERIEUR}

En dépit de leur privation de liberté, les détenus conservent le droit de communiquer avec leurs familles, leurs amis et consort. De ce fait, l'article 74 de l'ordonnance $344 \mathrm{du} 17 \mathrm{sep}$ tembre 1965 dispose : «Les détenus peuvent recevoir des visites aux jours et heures fixés par le règlement d'ordre intérieur, moyennant une autorisation spéciale du gardien. Sauf décision contraire du magistrat instructeur, les visites aux prévenus peuvent être autorisées. Le gardien ou un surveillant doit assister à ces visites.

L'autorisation du gardien n'est pas requise pour les visites du conseil du détenu. Celuici communique librement avec son client pour autant qu'il ait été régulièrement choisi ou désigné et qu'il agisse dans l'exercice de ses fonctions.

A moins de dérogations motivées par l'urgence, ces visites doivent avoir lieu aux jours et heures fixés par le règlement d'ordre intérieur ${ }^{1}$.

L'ensemble des principes pour la protection de toutes les personnes soumises à une forme quelconque de détention ou d'emprisonnement prévoit que « toute personne détenue ou emprisonnée a le droit de recevoir des visites, en particulier des membres de la famille, et de correspondre, en particulier avec eux, et elle doit disposer des possibilités adéquates de communiquer avec le monde extérieur, sous réserve des conditions et restrictions raisonnables que peuvent spécifier la loi ou les règlements pris conformément à la loi $»^{4}$.

Une atténuation particulière doit être apportée au maintien et à l'amélioration des relations entre le détenu et sa famille, lorsque celles-ci sont désirables dans l'intérêt de deux parties. Quelques implications à tenir en compte sont :

- L'emprisonnement aura souvent pour effet de provoquer l'éclatement des liens familiaux et la rupture des liens avec la communauté.

- Si les hommes et les femmes réussissent à maintenir des liens avec leurs familles, amis et la communauté pendant qu'ils sont en prison, cela limitera les effets préjudiciables de l'emprisonnement et facilitera vraisemblablement leur réintégration dans la communauté après leur libération.

Le maintien de ce contact est dans l'intérêt de tous :

* Les prisonniers seront en mesure d'assurer davantage la responsabilité de leur comportement, comme de leurs affaires personnelles et de se préparer à leur mise en liberté.

* Le travail du personnel pénitentiaire sera plus varié et plus satisfaisant, et la population carcérale dont ils ont la charge, sera moins inquiète de son avenir

* Les familles des prisonniers pourront offrir et recevoir un soutien précieux, dans l'immédiat comme par la suite, au lieu de perdre progressivement contact. 
* La communauté risquera moins de subir les nouveaux crimes si les prisonniers conservent leurs liens familiaux et si les projets ont été établis concernant leur activité qu'ils auront à leur sortie de prison ont été établis.

* Les mères emprisonnées ont des besoins spéciaux en matière de contact avec leur famille. Il faut toujours avoir présents à l'esprit les droits de leurs enfants (affection maternelle).

Le contact avec les familles est particulièrement important dans le cas des jeunes détenus. On dispose de cinq moyens principaux pour maintenir le contact avec le monde extérieur : Les lettres, visites, téléphone, libération conditionnelle et permissions de sortie, livres, les journaux et les médias.

L'essentiel à retenir est que les moyens énumérés ci-dessus seront d'autant plus facile à mettre en œuvre que l'organisation du système pénitentiaire permet de détenir les prisonniers dans des établissements carcéraux proches de leur domicile. Tout contact avec le monde extérieur est susceptible de poser certains problèmes de sécurité. Il importe donc de trouver des moyens permettant de maintenir des niveaux de contact appropriés sans compromettre la sécurité. Cela sera généralement possible, à condition que le personnel de la prison réalise que la sécurité et le contact avec le monde extérieur contribuent autant au succès de leur tâche délicate.

Les détenus reçoivent des visites, des lettres, ils échangent parfois avec leurs familles au téléphone, etc. un paragraphe du règlement d'ordre intérieur de la prison retrace tous les détails y relatifs, aussi différents textes appuient cet aspect si important. Les détenus reçoivent des visites aux jours et heures fixés par la police de ce service, c'est-à-dire : Mercredi et vendredi de 9 heures 30' à 17 heures 00 et Dimanche et jours les fériés de 9 heures 30 ' à 17 heures 00

Tout ce que nous avons dit concernant l'infraction de concussion commise chaque jour et à tout moment par les militaires et policiers commis à la sécurité de la prison de Kamina qui exigent une modique somme d'argent pour autoriser la remise de la nourriture aux détenus s'applique mutatis mutandis concernant les visites des détenus sauf celles effectuées par les activistes des droits de l'homme, les avocats et les défenseurs judiciaires.

Les pensionnaires de la prison de Kamina reçoivent et expédient des correspondances après avoir été censurées au préalable par le gardien de la prison. Notons aussi les visites des ministères de culte suivant l'horaire établi ci-dessous par le même règlement : Mercredi, c'est l'Eglise 30 ${ }^{\text {eme }}$ communauté pentecôtiste du Congo, de 7 heures $30^{\prime}$ à 9 heures 30'; Vendredi, l'Eglise Méthodiste unie, de 15 heures à 16 heures 30'; Samedi, aumônerie protestante, de 14 heures 30' à 16 heures 30'; Dimanche, Church of God, de 7 heures 30' à 9 heures 30 '.

Nous ne pouvons passer outre les visites des acteurs judiciaires tels que les magistrats du parquet de grande instance et auditorat militaire de garnison de Kamina et les avocats et défenseurs judiciaires. Les visites de différentes personnalités de bonne volonté, et celles des organisations non gouvernementales sont enregistrées. 


\section{II.6. DROIT AU TRAVAIL}

L'article 64 alinéa 1 de l'ordonnance 344 du 17 septembre 1965 sur le régime pénitentiaire dispose : « Le travail est obligatoire pour les détenus des prisons et camp de détention $»^{1}$.

Le travail pénitentiaire a longtemps occupé une position clé dans le bon fonctionnement des prisons. Il a fallu attendre la fin de la seconde guerre mondiale avant que le mouvement d'humanisation se traduise, sous l'influence de la défense sociale nouvelle, en une véritable politique d'amendement et de reclassement social des détenus ${ }^{10}$.on croit également à une retombée économique du travail. Les détenus ont la possibilité d'exercer des petites et grandes taches au sein de la prison, telles que l'entretien du cadre de vie carcérale (nettoyage des cours, balayage des couloirs...), les travaux champêtres..., ce qui permet d'obtenir un peu d'argent. Dans la prison de Kamina, ces emplois ne sont pas proposés à n'importe qui. En effet, il faut que le détenu qui les exerce soit digne de confiance, car celui qui sort les poubelles pourrait être tenté de s'évader de la prison et même celui qui travaille dans le champ parce que la prison n'est pas clôturée. La sélection des détenus est focalisée sur le comportement, le degré d'amendement et la peine de détenu. Les raisons pour lesquelles le législateur a imposé le travail à ceux qui exécutent une peine privative de liberté sont multiples. Elles tiennent à ce que le travail pénitentiaire revêt plusieurs aspects, dont l'aspect humanitaire, l'aspect rééducatif et l'aspect ré-adaptateur. Il est incontestable que l'inactivité est déprimante et que le travail constitue pour le condamné, un dérivatif nécessaire au maintien de son équilibre physique et psychique. Si les détenus n'ont pas du travail et s'ils s'habituent à l'oisiveté, ils risquent de perdre le sens de leur responsabilité vis-à-vis d'eux-mêmes et de leurs familles. Ainsi risquent-ils de trouver plus difficile de mener une existence respectueuse de la loi après leur libération. Quand il n'ya pas de travail pour les détenus, le personnel pénitentiaire doit être créatif et trouver d'autres moyens de les occuper.

Dans la prison de Kamina, l'organisation du travail pénitentiaire et le mode d'exploitation de la main d'œuvre pénale posent des problèmes particuliers, tant du point de vue matériel que juridique. Du point de vue matériel, il faut disposer du matériel approprié à chaque travail, des outils, machines et des infrastructures adéquates. Du point de vue juridique, il faut une loi pouvant détailler les modalités de travail, régler les devoirs, les obligations des détenus, fixer le taux de rémunération et la protection sociale du détenu travailleur. Les travaux dans les champs de la prison de Kamina sont organisés, durant toutes les deux saisons, c'est-à-dire la saison de pluie c'est la culture de maïs, haricot, arachide, courge..., et pendant la saison sèche, c'est la culture maraichère au profit des détenus, donc les choux de chine et pommé, le céleri, les tomates, les aubergines des carottes... cette activité est soutenue par la section pénitentiaire de la mission d'observation des nations unies pour la stabilité de la paix en République Démocratique du Congo. Le constat fait est qu'avec peu de matériel disponible et un minimum d'encadrement par le personnel pénitentiaire, le travail formel peut s'exécuter au grand bénéfice des détenus. 
L'article 69 alinéas de l'ordonnance 344 du 17 septembre 1965 dispose : « Le ministre de la justice du gouvernement central peut arrêter que les détenus des prisons ou des camps de détention bénéficieront pour chaque journée de travail, d'une allocation dont sept dixièmes seront affectés à la constitution d'un pécule disponible et trois dixièmes restent affectés à la constitution d'un pécule de réserve destiné à leur être remis après leur libération $»^{1}$. Le pécule est une remise d'une somme d'argent à un détenu à sa sortie de la prison, pour rémunérer le travail qu'il a effectué pendant son incarcération. La grande importance de cette allocation est le paiement soit de frais de justice, soit des amendes, soit encore des dommages et intérêts... Selon les sources pénitentiaires de Kamina, un ancien détenu qui a eu à faire le potager, avec les produits de la vente, il s'est payé un poste radio, meilleur souvenir qu'il garde de la détention.

Ce qui est regrettable dans la prison de Kamina, c'est le manque d'infrastructures de base (ateliers de tout genre), l'insuffisance de la sécurité, le risque d'évasion font que les prisonniers ne travaillent pas suffisamment.

\section{CONCLUSION}

Notre travail portant sur du respect des droits fondamentaux de l'homme dans la prison de Kamina est divisé en deux points. Dans le premier point, il est question avant d'entrer dans le fond de notre travail, de placer un mot sur la présentation, l'historique et le fonctionnement de la prison de Kamina. Le deuxième point quant à lui est consacré à l'état de lieu approfondi de droits des détenus dans la prison de Kamina. Il est divisé en plusieurs sous points. Le premier sous point traite le droit aux bonnes conditions d'incarcération des détenus. Le deuxième sous point analyse le droit à l'habillement décent des détenus. Le troisième sous point exploite le droit aux soins médicaux des détenus. Le quatrième sous point se focalise sur les droits à une nourriture équilibrée et suffisante des détenus. Le cinquième sous point s'appesanti sur les droits des détenus aux contacts avec l'extérieur et le dernier sous point se basé sur le droit au travail des détenus.

Nous avons amèrement détaillé tout au long de notre travail comment tous les droits prévus par l'ordonnance 344 du 17 septembre 1965 sur le régime pénitentiaire en faveur des détenus de la prison de Kamina ne sont pas respectés à cause des infrastructures et des finances. Le bâtiment abritant la prison de Kamina n'est pas approprié pour cette fin. Les conditions de détention, sanitaire, de restauration et sécuritaire se posent avec acuité car les conditions de vie des détenus sont précaires, humiliantes, dégradantes et avilissantes (surpopulation, promiscuité, prolifération des maladies...). Les pensionnaires de cet édifice ne sont soumis ni à l'enseignement, ni à la formation professionnelle ou encore aucune mesure de grande envergure n'est amorcée dans le sens de la réinsertion. Celle ci est un processus amorcé en principe dès la détention. C'est une prise en charge qui nécessite les moyens financiers adéquats et le concours de tous, mais pour la prison de Kamina l'Etat ne s'y intéresse pas afin d'améliorer les conditions de vie et celles du processus de la réinsertion des détenus. 
Notre travail sera dépourvu d'un intérêt pragmatique si nous n'y mentionnons pas quelques suggestions pour améliorer les droits des détenus de la prison de Kamina. De ce qui précède, nous suggérons ce qui suit :

- Que le gouvernement central de la République Démocratique du Congo construise une nouvelle prison à l'écart de la ville de Kamina en respectant les normes internationales requises en la matière. En outre, qu'il l'équipe en matériels indispensables tels que les lits, mousses, draps, couvertures, fer à repasser, équipements médicaux... et la desserve régulièrement en nourriture suffisante et équilibrée, désinfectants, produits pharmaceutiques, habillement décent... en faveur des détenus.

- Que les contrôles de la prison par l'inspecteur provincial chargé de la section d'inspection des établissements pénitentiaires, le gouverneur de la province du Katanga ou son délégué, L'administrateur du territoire de Kamina, le médecin désigné par le gouverneur de la province, l'officier du ministère public Soient effectués régulièrement.

- Au législateur, de voter une loi règlementant le travail en milieu carcéral et instituant le travail d'intérêt général dans notre droit concernant les condamnés à au plus une année de servitude pénale.

- Aux agents pénitentiaires, de prendre leur travail au sérieux car la réinsertion du détenu dépend de la prise en charge du condamné par eux qui doivent tout mettre en œuvre pour que le temps d'emprisonnement constitue un temps socialement utile.

- Aux organisations non gouvernementales et à la société civile, de prêter main forte à l'administration pénitentiaire en créant un service d'assistance sociale qui pourra accompagner les anciens détenus de la prison de Kamina et d'aider ces derniers à s'adapter à la vie sociale après leur sortie de la prison.

\section{REFERENCES BIBLIOGRAPHIQUES}

1. Ordonnance 344 du 17 septembre 1965 portant régime pénitentiaire, Articles 9,49-50, 48,51-52, 59 alinéa 1, 59 alinéa 2, 54 alinéas 2-3, 55 alinéas 2-3, 60, 61 alinéa 1,62 alinéas 1-2, 74, 64 alinéa 1, 69 alinéa 1

2. Comité international de la croix rouge, Hygiène et habitat dans les prisons, Genève, 2004, P.P.17, 20

3. KATWAMBA BASA MUNDA, Problématique de la validité de la peine et son impact sur l'amendement et la réinsertion sociale du délinquant en République Démocratique du Congo, Mémoire de diplôme d'études approfondies en Droit, Université de Kamina, Juin 2012, P.P. 116,117

4. Nations unies, les Droits de l'homme et les prisons, New York-Genève, 2004, P.P. 27,64

5. Rapport d'inspection du procureur de la République près le tribunal de grande instance de Kamina, Février 2012

6. Organisation non gouvernementale USAHIDIZI, Manuel de nutrition, Lubumbashi, 2006, P.16 
7. La constitution telle que modifiée par la loi n¹1/002 du 20 janvier 2011 portant révision de quelques articles de la constitution du 18 février 2006 de la République Démocratique du Congo, article 203 alinéa 1

8. AKELE ADAU et all, Notes polycopiées du cours de Droit pénal spécial, troisième graduat droit, Université protestante au Congo, 2003-2004, P. 255

9. Décret du 30 janvier 1940 tel que modifié et complété à ce jour, article 149

10. Http//Champ pénal.Révues.org/684 (Consulté le 28 décembre 2014) 